EDITORIAL

\title{
Multidisciplinary interventions in heart failure
}

\author{
A L Clark, J G F Cleland
}

Heart 2005;91:849-850. doi: 10.1136/hrt.2004.056242

\section{A National Service Framework for heart failure is urgently needed}

$\mathrm{D}$ octors working in the field of chronic heart failure are, in many ways, in an enviable position. They have a range of effective treatments that can approximately double life expectancy for patients with significant left ventricular systolic dysfunction ${ }^{1}$ and can dramatically reduce the need for repeated hospitalisations. Their decisions on treatment are based on a wealth of evidence from carefully conducted multicentre trials, and they can be in no doubt of their potential worth to their patients. In their practice, they are supported by a profusion of available guidelines produced by experts who have carefully sifted the evidence. Technological advances, such as biventricular pacing and implantable defibrillators, expand the range of treatment options.

Despite this, the evidence suggests that the research effort has not resulted in universal uptake of adequate investigations and treatment and management of heart failure patients. The Euroheart survey ${ }^{2}$ gives a snapshot for considering the current state of practice. In a panEuropean study of over 11000 patients admitted acutely to hospital with heart failure, only two thirds had ever had an echocardiogram. An angiotensin converting enzyme (ACE) inhibitor was prescribed to $62 \%$ of patients and a $\beta$ blocker to just $37 \%$. Only $17 \%$ were on the combination of diuretic, ACE inhibitor, and $\beta$ blocker.

This state of affairs matters greatly to the individual patient, whose life chances are being reduced, but also to society more broadly. Heart failure is an enormous consumer of health care resources. The largest proportion of those costs is on inpatient hospital care, and heart failure patients have an extremely high re-admission rate.

\section{DELIVERY OF CARE}

The contrast between what we know is achievable and what we know is happening suggests a systemic problem with the delivery of care to heart failure patients. Despite the difficulty in supporting trials of non-pharmacological management approaches, the report in this issue by Holland and colleagues ${ }^{3}$ shows that there is a way forward. Structured management programmes that include patient and carer education, the ready availability of advice and support, and, in particular, some home visits, improve the life expectancy of patients and reduce their need for repeated hospitalisations.
Present models of healthcare delivery are mainly reactive and focused on the relation between patient and doctor. The patient perceives a problem, and makes an appointment to see the doctor. Medicine is then done to the patient in the hospital or surgery. The challenge posed by the results of analyses like Holland's is that we need to re-think this model, at least for heart failure care. Reaction is not the right approach: patients need to be able to become active partners in their management, and the management strategy needs to be structured to provide continuing care and support.

Changing the model is of particular importance for people with chronic disease. Chronic heart failure is a systemic disease caused by cardiac dysfunction and it is complex to manage both cross sectionally (patients may have anaemia, renal dysfunction, cardiac dyssynchrony, myocardial hibernation, associated valve disease, cardiac arrhythmia, and diabetes to mention but a few) and longitudinally (the disease is not static and tends to evolve and therefore requires regular expert review).

The role of nurses as practitioners in their own right, and the role of pharmacists and physiotherapists have to be recognised. There is some formal recognition of the possible worth of "heart failure nurses" with enthusiastic development of their roles in some areas. Uptake is, however, patchy, and depends in large part on the enthusiasm of a heart failure specialist in a local hospital. In many parts of the UK there are simply not enough cardiologists to serve the population, and certainly no heart failure cardiologist to support a demanding new role.

\section{NATIONAL SERVICE FRAMEWORK}

Government initiatives, such as the National Service Frameworks (NSFs) and guidance from the National Institute for Clinical Excellence, have proved the engine for forcing through improvements in care for patients with coronary heart disease. These developments are admirable, but can have their downsides. The deliverers of health care have to "deliver targets" chosen centrally (often chosen because they are, at least, countable). Things not included in a framework document become lower priorities and can be sacrificed to some degree to meet the targets. Heart failure, of course, was not a central feature of the NSF for coronary artery disease. It appears as chapter 6, and does contain milestones for primary care and targets for secondary care. By April 2002, "clinical audit data no more than 12 months old that describe the delivery of the key investigations and treatments" should be available. The stated NSF goal is that "every hospital should: offer complete and correct packages of 
audited effective interventions to all people discharged with a diagnosis of heart failure, demonstrated by clinical audit data no more than 12 months old." It is difficult to think of many cardiac networks where these aspirations have been realised. Management structures seem to be able to put heart failure to one side.

Heart failure care remains a Cinderella of cardiology. Although the advent of device therapy has given a veneer of glamour to proceedings, there is often no systematic approach to overall management. It can be a matter of chance who is referred for device therapy, and once the device is implanted, there is, in many places, no heart failure cardiologist available to take on continuing care. Devices can have a striking beneficial effect for some patients, but their overall impact is small in comparison to the potential effect of adequate delivery of medication. Device therapy disproportionately engages administrators and guideline producers.

We should be engaging with the failure to provide much more straightforward care. What is needed is recognition by central government that heart failure is common and expensive but treatable. A National Service Framework for heart failure with specific targets is a priority. To whet a politician's appetite, we can even say with some certainty that it might save money.

\section{Authors' affiliations}

A L Clark, J G F Cleland, Castle Hill Hospital, Castle Road, Cottingham, Hull, UK

\section{REFERENCES}

1 Cleland JG, Clark AL. Delivering the cumulative benefits of triple therapy to improve outcomes in heart failure: too many cooks will spoil the broth. J Am Coll Cardiol 2003:42:1234-7.

2 Komajda M, Follath F, Swedberg K, et al, for the Study Group on Diagnosis of the Working Group on Heart Failure of the European Society of Cardiology. The EuroHeart Failure Survey programme-a survey on the quality of care among patients with heart failure in Europe. Part 2: treatment. Eur Heart $J$ 2003:24:464-74

3 Holland R, Battersby J, Harvey I, et al. Systematic review of multidisciplinary interventions in heart failure. Heart 2005;91:899-906.

\section{IMAGES IN CARDIOLOGY}

\section{Spontaneous triple vessel coronary artery dissection in a patient with effort angina}

S pontaneous coronary artery dissection SCAD) is a rare but often fatal cause of schaemic heart disease occurring predominantly in young or middle aged, otherwise healthy subjects. Although about 250 cases of SCAD have been reported in the literature, to the best of our knowledge, spontaneous three vessel coronary dissection associated with typical effort angina has never been reported. This case illustrates coronary angiography and intravascular ultrasound images in a patient with SCAD involving all three vessels and treated by stenting.

A 57 year old man presented with exertional chest pain. His risk factors included smoking, hypertension, and hyperlipidaemia. ECG showed an abnormal Q wave in lead III and $1 \mathrm{~mm}$ ST depression in III, aVF. Technetium-99m sestamibi SPECT image showed a moderate fixed defect in the inferior wall and a reversible defect in the anterior wall. Selective coronary angiography revealed SCAD involving all three vessels. Right coronary angiogram showed linear dissection starting from the ostium associated with distal total occlusion (panel A). Intravascular ultrasound (IVUS) revealed prominent dissection flap with false lumen $\left({ }^{*}\right)$ (panel B). After successful angioplasty and stenting, no visible dissection flaps with full recovery of true lumen were seen (panel C). SCAD at left anterior descending artery (panels D-F) and left circumflex coronary artery (panels G-I) were also successfully treated by stenting after assessment with IVUS. Five days after intervention, the patient did not complain of chest pain and was discharged.
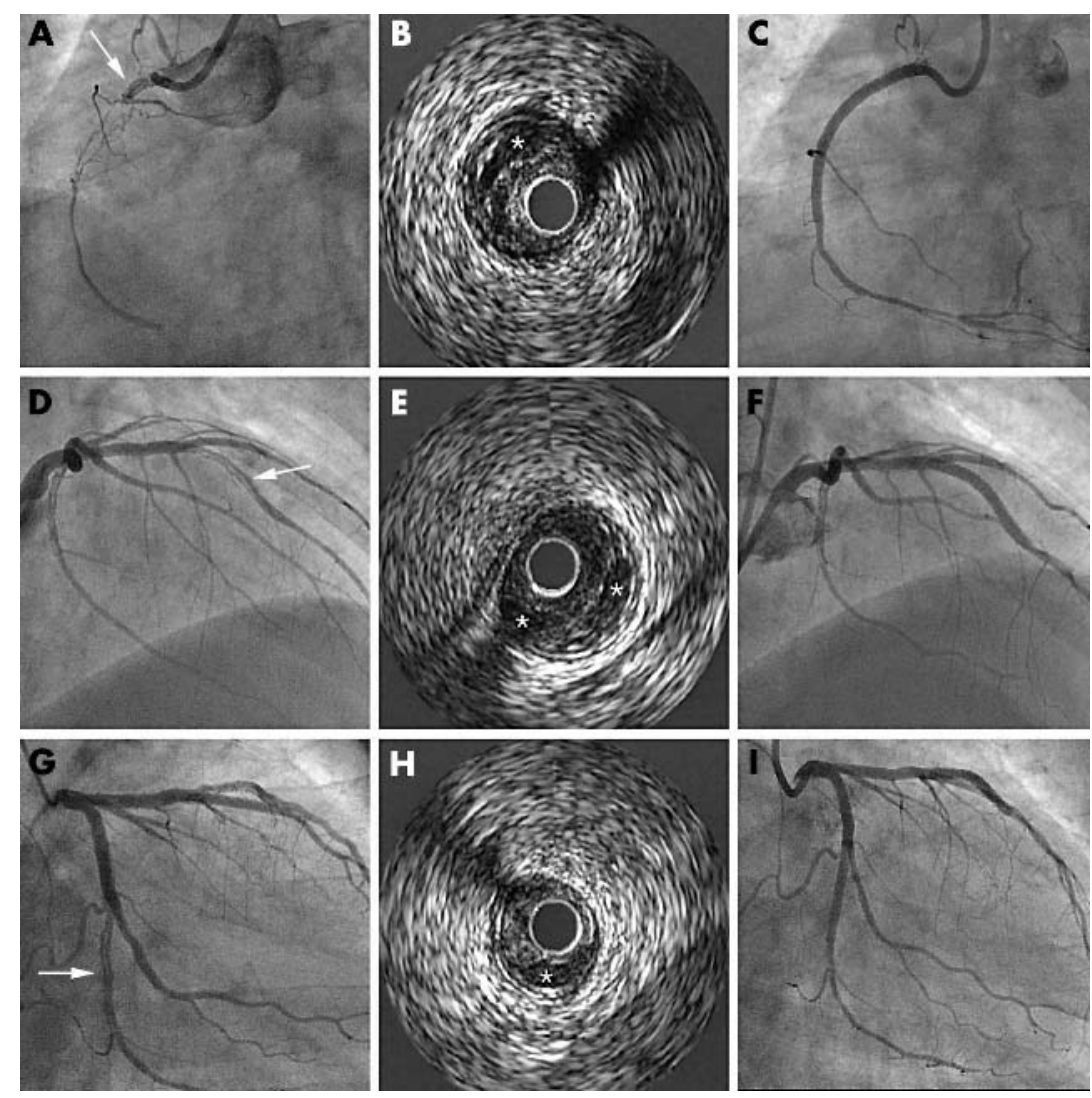

E-Y Choi 